\title{
Das Alter ist ein begleitender Freund
}

\section{Elisabeth Seifert}

Chefredaktorin der Fachzeitschrift Curaviva

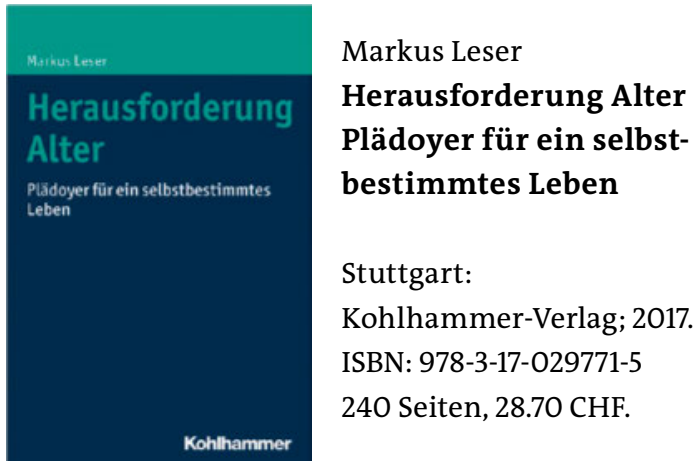

Seit 30 Jahren beschäftigt sich der promovierte Gerontologe Markus Leser mit Fragen rund um das Thema Alter. In einem persönlich geschriebenen Buch gibt er jetzt Einblick in seine Erkenntnisse, Erfahrungen - und Forderungen: Es sei die Aufgabe der Gesellschaft, den Stillstand und die Tabuisierung des Alters, gerade auch des hohen Alters und des Sterbens, aufzulösen und sich für ein differenziertes Altersbild einzusetzen. Besonders in die Pflicht nimmt der Autor seine eigene Generation, die Babyboomer. Erstmals in der Geschichte macht mit ihnen ein so grosser Teil der Bevölkerung die Erfahrung des Älterwerdens. In ihrem eigenen Interesse muss es deshalb liegen, eine eigentliche Alterskultur zu entwickeln. Dies bedingt einen Perspektivenwechsel: Das Altern dürfe nicht als Verhängnis oder ein zu bekämpfender Feind wahrgenommen werden, sondern vielmehr als ein begleitender Freund.

Eingebettet in dieses zentrale Anliegen des Buches erörtert Markus Leser, seit 2003 Leiter des Fachbereichs Menschen im Alter von Curaviva Schweiz, eine Vielzahl von Aspekten rund um das Alter und das Altwerden. Eine kleine Kulturgeschichte des Alters gehört genauso dazu wie eine kritische Würdigung moderner Technologien, die im Alter Entlastung bringen sollen. Besonders lesenswert ist das Kapitel zur Enttabuisierung des Lebensendes. Es sei fahrlässig, wenn die mediale Debatte über Sterbehilfe auf die Frage des begleiteten Suizids fokussiert, so seine Kritik. Eine umfassende Sterbebegleitung, wie sie die Palliative Care leisten könne, werde viel zu wenig thematisiert. Auch krankes Leben ist lebenswert und lässt sich wertvoll gestalten, betont der Gerontologe. Unsere Gesellschaft müsse das Sterben als Teil des Lebens wahrnehmen und alle vom Sterben Betroffene auf würdevolle Art und Weise unterstützen. Damit dies gelingen kann, brauche es eine noch bessere interdisziplinäre Zusammenarbeit zwischen Pflegenden und Ärzten. Breiten Raum nehmen im Buch die Themen Wohnen und Pflege im Alter ein sowie die Frage der Kosten und ihrer Finanzierung. Er stimmt dabei nicht ein in das öffentliche Lamentieren über die steigenden Ausgaben infolge des demografischen Wandels. Die Gesellschaft müsse sich zunächst darüber klarwerden, welches Angebot sie für pflegebedürftige alte Menschen bereitstellen will. Erst in einem zweiten Schritt mache es Sinn, die Kosten für dieses Angebot auszuweisen und über die Finanzierung nachzudenken. Eine Vielzahl von Studien mache dabei längst deutlich wohin die Entwicklung im Wohn- und Pflegebereich gehen muss. Gerade die Generation der Babyboomer fordere die Anerkennung von Autonomie und Selbstbestimmung bis ins höchste Alter. Alterspflegeinstitutionen, die diesen Trend erkennen, verstehen sich deshalb zunehmend als Dienstleistungsunternehmen, die auch pflegebedürftigen betagten Menschen ein selbstbestimmtes Leben in der von ihnen bevorzugten Wohnumgebung ermöglichen. Eine ganze Reihe stationärer Einrichtungen in der Schweiz haben sich in diesem Sinn bereits auf den Weg gemacht, wie Markus Leser betont. Ein Gebot der Stunde ist die Kombination von Pflegeinstitutionen und betreuten Alterswohnungen. Konsequent umgesetzt wird die Idee des Heims als Dienstleistungszentrum im Wohn- und Pflegemodell 2030 von Curaviva Schweiz. Das Modell ermöglicht dabei eine Kooperation verschiedener Dienstleistungsanbieter und setzt neben Profis auch auf die Mitarbeit von Nachbarn und Freiwilligen. Gefragt ist ein Miteinander von Organisationen und Personen innerhalb einer bestimmten Region.

Ein Gewinn ist das Buch von Markus Leser nicht zuletzt durch die vielen Literaturzitate, die weit über die eigentliche Forschungsliteratur hinausgehen. Eine leicht verständliche Sprache macht es zudem für Interessierte ausserhalb des engeren Adressatenkreise zugänglich. In erster Linie richtet sich das Werk an Gerontologen sowie Fach- und Führungspersonen in der ambulanten und stationären Pflege und Betreuung. 